\title{
SOME PAINFUL CONDITIONS ABOUT THE ANUS.
}

\author{
BY ASLETT BALDWIN, F.R.C.s. \\ Consulting Surgeon to St. Mark's, the West London, and Freemasons' Hospitals.
}

A CAKEFUL examination should always be made when a patient complains of pain about the anus. The home-made diagnosis is almost always "Piles," but it is often wrong.

Foreign Bodies.-A lubricated finger gently passed into the bowel may feel a fish-bone or other foreign body. When one is found it is well to inject a surface anæsthetic into the bowel such as percaine. After a few minutes the finger may be able to hook out the offending object. If it is lying transversely, pressure on one side of the bowel with the examining finger may enable it to be dislodged into the vertical position and its removal made easy. If the body cannot be dislodged, perhaps it could be divided with bone forceps ; if unsuccessful, an anæsthetic should be given, low-spinal or ether (not chloroform), the sphincters dilated and the bowel washed out with a non-poisonous antiseptic, before further attempts at removal. Various specula and forceps may be required. After removal an antiseptic suppository, such as iodex, should be placed in the bowel, to try and prevent infection of any punctures or abrasions of the mucous membrane.

Cases are recorded in which a linear rectotomy backwards proved unavailing, and the abdomen was opened below the umbilicus with success.

Thrombotic External Piles.-These are rounded or oval swellings at the verge of the anus, usually varying in size from that of a pea to a small marble, deep red or bluish in colour. Pressure on the veins above, as from constipation or pregnancy, is the predisposing cause; the exciting cause is strain, often during defæcation. A sharp, pricking pain is noticed at the outset, followed by severe pain and tenderness. The lump is caused either by a clot in a dilated vein, or by rupture of a small vein into the connective tissues, forming a hæmatoma. I have seen both conditions. The pain is caused by tension and pressure on the sensitive nerve-endings.

The best treatment is to wash the skin with lysol, $3 \mathrm{j}$ to a pint, or carbolic lotion, I : 40. After drying with sterile absorbent wool, and painting with tinct. iodi, and allowing the spirit to evaporate, a spot of liquid carbolic acid is placed on the skin about half an inch from the swelling, and after about two minutes 2 per cent. novocain with adrenalin is injected with a very fine needle, starting where the skin has been made insensitive by the carbolic. The fluid should be slowly injected on both sides of the swelling and under it, the needle being advanced into each anæsthetic area and should not be completely withdrawn till all the injections are made. The pile is then seized with toothed forceps and two-thirds of it cut off with scissors. If the clot is in a vein it easily slips out, if it is a hæmatoma the blood may look like bits of black cotton, and pointed scissors may be required to remove it and the tissue into which it has extravasated. The parts must be kept very clean and the wound powdered at first with borochloretone, and in about twenty-four hours with aristol, with a little anæsthesin added if necessary. A small piece of white lint placed in position will generally remain there.

If the patient will not submit to this little operation, the lump will usually subside slowly with hot fomentations of lead and opium, or glycerin of belladonna, but it usually leaves a skin tag, which may cause trouble afterwards, or it may suppurate, necessitating an incision with removal of skin, to avoid a fistula. 
All wounds about the anal region heal much better if the patient is kept as much as possible in the recumbent position, because there are no valves in the veins between the heart and the hæmorrhoidal veins.

\section{Anal Fissure.}

This is an extremely painful affection and demands great gentleness during examination and treatment. Pain is acute while the bowels are acting and may be severe for hours afterwards, which often causes the act to be put off for days and when the bowels do act the patient may be completely prostrated.

In an early case it looks like a crack or fissure in the mucous membrane, generally situated posteriorly, sometimes anteriorly, rarely to one or both sides; if multiple, syphilis should be suspected. Later on it is an ulcer, with nerve-endings exposed in its floor ; irritation of these causes the sphincters to be tightly contracted, which may cause the fissure to be completely hidden. As the patient may be in terror of being touched, a local anæsthetic must be carefully applied, an exploration with the finger may be out of the question.

The patient being on the left side, a little cotton-wool is twisted round the end of a probe and moistened with water, then dipped into anæsthesin powder and gently introduced down the opposite side of the bowel to the fissure and allowed to remain for a short time and then gradually pressed backwards towards the ulcer. When anæsthesia is produced, the buttocks may be gently separated and the fissure brought into view. It will be seen if there is a "sentinel pile" at its lower end, this is an odematous tag of skin and shows that the fissure is not a recent one. When anæsthesia is sufficient, ac finger lubricated with "lubafax" with anæsthesin powder, should be gently inserted" into the anus, the pulp of the finger towards the fissure, its back pressing away from the fissure. By this means it will be ascertained if there is a polypus at its upper end, if its edges are indurated and inspection may perhaps reveal fibres of the external sphincter in its floor.

The treatment I prefer and which gives excellent results in cases which are not too old and complicated, is to inject urea and quinine under and perhaps each side of the fissure, the former is often sufficient.

A two-grain tablet is dissolved in 45 minims of water in a test-tube and boiled just before use, the extra 5 minims are to allow for evaporation. About 20 minims of this are injected under the fissure, with the same precautions as described for thrombotic piles, but the fluid must be injected very slowly indeed and directly the patient complains of pain stop the injection and only proceed when told that the pain' has gone. A quick injection is extremely painful, a comfortable one may require ten or thfteen minutes. The fissure should then be touched with pure ichthyol. The patient should use a sedative ointment such as hazeline, with 2 per cent. anæsthesin, if necessary by means of an ointment introducer, before and after each action of the bowels and at night. A suppository may be used containing ichthyol 3 gr., anæsthesin 2 gr., ol. theobrom q.s. provided it is just introduced into the anal canal and supported there by a finger until partially dissolved, if allowed to go into the rectum at once it would be too high up to be of much use.

The motions must be kept quite soft by taking a teaspoonful of paraffin three or four times daily as necessary to get a soft stool once daily. 
The parts must be kept scrupulously clean, sitting in a hot bath with each ischial tuberosity on a half brick or similar support is grateful and comforting.

When the sphincters are very tight and indurated, much benefit is obtained by stretching them under a general or low spinal anæsthetic, the patient being in the lithotomy position supported by Clover's crutch. Chloroform should not be used.

The patient should be seen at least twice weekly by the Doctor, who should apply ichthyol to the sore by means of cotton-wool wrapped round the end of a probe or through a small speculum with a side slit.

In some chronic and complicated cases it will be advisable to operate.

The patient being anæsthetized with ether or low spinal anæsthesia and in the lithotomy position, the rectum is washed out with a non-poisonous antiseptic, the sphincters dilated, and the rectum dried with swabs. A suitable speculum is introduced and a careful examination is made. There may be a polypus or enlarged anal valve at the upper end of the fissure. A probe bent into a hook should explore the anal crypts or lacunæ just at the top of the anal canal. There may be ulceration there or a small fistula leading from it, perhaps extending under the fissure. Enlarged papillæ should be well crushed with Spencer-Wells' forceps to avoid bleeding and cut off. An incision is then made with a sharp knife starting at the top of the fissure, and being gradually deepened till it is about half an inch deep well out in the skin, the skin part should be twice as long as that in the anal canal. This is to prevent the skin part of the incision from healing before the anal part and so insure good drainage. As a rule no part of the sphincter needs division. The edges of the skin and of the fissure are removed with sharp-pointed scissors, also the sentinel pile or cedematous tag of skin at the lower end of the fissure; any granulations are removed with a Volkmann's spoon and the fissure lightly touched with liquid carbolic. A small piece of wool soaked in sterile olive oil or vaseline is laid on the wound and should extend a little way up into the bowel above the fissure. This should be renewed twice daily and not packed tightly into the wound. The patient should be kept lying down as much as possible, or healing will be delayed. Great cleanliness must be observed, and the rest of the after-treatment is similar to that when no operation has been performed.

Some surgeons advocate complete excision of a fissure with skin below it in chronic and complicated cases, but this should seldom be necessary.

Irritable Ulcer of the Anal Canal.-This is usually situated dorsally and just above the usual situation of a fissure.

The symptoms are somewhat similar to those of the latter, but do not come on during the passage of a motion as in fissure, but some time afterwards, and the pain is not so severe as with fissure. The pain may radiate over the sacrum, or to the bladder or vagina, or down the thighs as in fissure, but as a fissure is not visible may be mistaken for neuralgia. The ulcer may be in a crypt, just above or behind an anal papilla, and there may be more than one. A speculum will be required to see it. They may not be easy to find.

The treatment consists in dilatation of the sphincters, crushing and snipping off any anal papilla or mucous membrane covering the ulcer. The former are very sensitive. The after-treatment is the same as for fissure.

For ulcers at the lower end of the rectum I have had very good results with zinc ionization. 
A pure zinc electrode is taken and about two inches of one end are wrapped round with several layers of white lint, round this is tied one layer of waterproof jaconet, with thread. The number of layers of lint depend on the size of the ulcer. A hole is cut in the jaconet, halfway down its length, somewhat larger than the ulcer; the lint is soaked in 2 per cent. zinc sulphate solution; a sprinkling of cocain powder is spread over the exposed lint. The jaconet is lubricated with lubafax, not vaseline, or the passage of the electric current may be prevented. The window of the jaconet is placed in contact with the ulcer. The electrode is attached to the positive pole of a battery. The negative pole is attached to a good-sized flat electrode, with about ten layers of lint under it soaked in 2 per cent. common salt solution; this is placed over the sacrum. If the ulcer is in the anterior part of the bowel the negative pad is placed on the lower abdomen. The current is turned on very slowly up to about ro ma. for 20 minutes or so, according to the patient's feelings, and slowly turned off. The cocain and zinc ions are driven into the ulcer and microbes are drawn out and killed. After several days the treatment is repeated if necessary. It is surprising how quickly this treatment will cause an ulcer to heal.

Abscess. - A superficial abscess is obvious, but a submucous or ischiorectal abscess before attaining a large size may easily be missed unless the index finger is introduced into the bowel and the tissues all round palpated between it and the thumb. It cannot be too strongly urged that an abscess, or one that is about to form, should be operated upon at once, and not have expectant treatment by hot fomentations in the hope that it will "disperse" or burst itself. The former will not take place; the latter may do so but it will not be a cure, although the patient has temporary relief. The skin opening will close before the abscess cavity has healed; pus will reaccumulate, and if treated in the same way will burrow and form a fistula. Delay causes loss of time, much suffering, and one or more operations before a cure is achieved.

If the operation is a small matter, it may be done with a local anæsthetic, novocain 2 per cent. with adrenalin; there are several other good anæsthetics in use.

If somewhat larger, gas and air or oxygen will suffice, but precautions must be taken to keep the patient in the required position or he will suddenly extend the hip- and kneejoints and nothing can be done. A Clover's crutch will hold him in the lithotomy position, or, if on the side, a strong jack towel should be placed round the knees and slipped over the neck. The towel will be too long and will require to be shortened by being tied round with thick string or thin rope.

If the abscess is of any size, a low spinal or general ether should be employed, especially if the patient is a strong person or very nervous.

With the patient in the lithotomy position, the parts are washed with a solution of lysol, dried, and painted with tincture of iodine. An incision radiating from the anus is made right along the abscess and into the skin beyond it, and another at right angles to it right across the centre of the abscess, forming a crucial incision. Four pointed pieces of skin will now be formed ; two-thirds or more of these must be cut away with scissors, the skin being held by the tips with toothed forceps. The amount of skin to be removed depends on the depth of the abscess. The pus being evacuated, a finger is introduced into the cavity and any fibrous septa broken down and all the loculi of the abscess converted into one cavity. It is not necessary to irrigate for several days, but if there is a large abscess it may be advisable to do so to ascertain if pus is coming down from above 
the levator ani. If that is found, the tract should be enlarged by Hilton's method, care being taken not to open the peritoneum, and a good-sized drain-tube inserted and secured by stitches.

An ointment such as lanocyllin, containing a surface anæsthetic, anæsthesin, 2 per cent., pantocain, 2 per cent., orthoform, 3 per cent., or cycloform ointment, should be kept smeared along the cut edges of the skin. This will prevent pain and tenderness and prevent the dressings from sticking and make the patient much more comfortable. Hot fomentations are applied four-hourly, and, if possible, the patient should sit in a hot bath containing some non-poisonous antiseptic for about twenty minutes once or twice daily. When the discharge has almost ceased, the dressing can be gauze made damp with I : I,000 flavine and, finally, the wound can be dusted with aristol and covered with dry gauze and wool.

If the abscess contains gas or surgical emphysema is present, anti-gas-gangrene serum should be injected. Adrenalin chloride solution $\mathrm{I}: \mathrm{I}, 000$ should be at hand and $10 \mathrm{~m}$. given hypodermically in case of anaphylaxis.

In an early case no pus may be found, but a crucial incision in the indurated area will do nothing but good and may prevent abscess formation, and, combined with hot fomentations, pain. will soon be relieved.

If a fistula has already formed, it should not be operated upon at the same time as an acute abscess, but left for several weeks, when the resulting wound will be much smaller. In some cases, when a large granulating surface is left, Mr. Gabriel has successfully employed skin grafting. "Thiersch grafts are applied in the usual manner and left exposed to the air without any dressing. Warm saline solution is dropped on them every hour or two for the first few days, and when they have 'taken' a stimulating dressing of brilliant green solution I : I,000 can be applied on gauze. The bowels must be confined for five to seven days after the skin-grafting" (vide "The Principles and Practice of Rectal Surgery," by Wm. B. Gabriel. H. K. Lewis and Co. Ltd., London).

In all the cases referred to, the patients should be kept in the best possible hygienic condition, and as most likely their food has been deficient in vitamins these should be given freely. Wounds which are at first very sluggish will soon start to heal quickly.

Vitamin compound made by Allen and Hanburys contains A, B and D. Haliver oil. or adexolin capsules, \&c., for $A$ and $D$ vitamins ; bemax or yeast or phyllosan for $B_{1}$ and $\mathrm{B}_{2}$; orange, lemon or grape-fruit juice for $C$. For the best results, all the vitamins should be supplied.

Parathyroid tabloids (desiccated) $\frac{1}{1}$ ogr. twice daily, are very useful to promote healing, as with leg and other ulcers.

\section{CASES DEMONSTRATED AT THE M.R.C.P. CLASS.}

\section{A Case of Spontaneous Pneumothorax.}

O. W., female, aged 8.

Admitted to hospital, April, I932. Complained of pain in the abdomen. This pain gradually got better, and in August the child was sent to a convalescent home, where she remained till February, 1933. During this period she failed to put on weight, and has now been discharged.

Past history and family history are negative.

This case was put up for diagnosis. 